\title{
Forensic Odontology: An Aid in Identification of Unknown Human Remains
}

\author{
Hemlata Pandey ${ }^{1}$, Sumit K. Chaudhary ${ }^{2}$, Harish Pathak ${ }^{3}$, Emilio Nuzzolese ${ }^{4}$ \\ ${ }^{1}$ Research Scholar, ${ }^{2}$ Senior Assistant Professor, School of Forensic Science \& Risk Management, Rashtriya Raksha \\ University, India, ${ }^{3}$ Professor and Head, Department of Forensic Medicine, Seth GS Medical College and KEM \\ Hospital, India, ${ }^{4}$ Professor, Department of Public Health Sciences and Pediatrics, Section of Legal Medicine, \\ University of Turin, Italy
}

\begin{abstract}
The robust nature of teeth and their ability to resist destruction, make them indispensable evidence in cases where human identification by a scientific method is required. Forensic odontology is the branch of dentistry that combines knowledge of teeth and oral and maxillofacial region of a dentist with the legal system. Dental identification has proven to be a boon in disaster victim identification. Besides teeth, an odontologist can also help identify unknown domains from other features such as the palatal rugae, lip prints, frontal sinus, etc. Forensic Odontology can also assist in the biological profiling of human remains by sex determination, age assessment, racial profiling, and information about habits or pathologies from dental traits. Dental age estimation is a crucial part of the age estimation process in the medico-legal system. Research has shown that dental age estimation by studying mineralization and development of teeth in children and young adults can be fairly accurate and supplement skeletal age assessment. Biological profiling is required to be done for reconstructive identification by forensic facial reconstruction, especially in cold cases. Equipped with the expertise of dental structures, oral and maxillofacial region, overall skull anthropology, forensic odontology can contribute towards the identification of skeletonized, decomposed, or mutilated remains especially in cold cases.
\end{abstract}

Keywords: Forensic Odontology, dental Identification, dental age estimation, forensic facial reconstruction, cheiloscopy, rugoscopy, denture markers

\section{Introduction}

Forensic Dentistry, a term interchangeable with Forensic Odontology, is the area of dental practice encompassing the interaction of the dental team with the legal system, both civil and criminal. The field includes; but is not limited to, the identification of human remains; population/ ethnicity identification,

\section{Corresponding Author:}

Dr. Hemlata Pandey, Research Scholar, School of Forensic Science \& Risk Management, Rashtriya Raksha University, Lavad, Dahegam, Gandhinagar-382305, Gujarat, India

Email ID: hemlatapandey@gmail.com sex determination, age estimation, bite pattern analysis; person abuse, including child abuse, sexual assaults; personal injury, including malpractice, dental fraud; and disability estimation ${ }^{1}$. In addition to litigation, the discipline is concerned with research in the many areas encompassed by the field. Because teeth are highly resistant to destruction and their arrangement and characteristics are unique to the individual, they and their bite patterns provide evidence often beneficial in civil and criminal litigation. The ability to recognize, collect, store and preserve, organize, document, analyze, report, and present such information is the heart of forensic dentistry. 
Forensic Odontology, or forensic dentistry, has been defined by Keiser-Neilson in 1970 as "that branch of forensic medicine which in the interest of justice deals with the proper handling, examination of dental evidence and with the proper evaluation and presentation of the dental findings in criminal and civil proceedings ${ }^{2}$. It is not limited to one area of dental science; rather it strives to synthesize principles, knowledge, and competencies from many aspects of dentistry with those not found within the usual dental curriculum. Fields outside the scope of normal dental experience include photography, law enforcement procedures, forensic pathology, radiology, legal knowledge, and physical anthropology among others. Forensic dentistry is a multifaceted, interesting, and rewarding blend of dentistry and the law. For most who participate in the field of forensic odontology, there is no great financial reward, but the satisfaction of performing difficult and challenging tasks well is immensely rewarding. A forensic odontologist's work can have a great impact on the lives of individuals and families.

\section{Dental Identification of Human Remains}

When human remains are found, the priority of the investigation is to ascertain the identity of the deceased; indeed, any forensic investigation involving human remains would be very difficult to solve without this information. Several methods and techniques from diverse fields, depending on the remains available, can be applied to human identification. The first step in the identification process is to build up a biological profile, which is a general description of the individual's ancestry, sex, age-at-death, and stature. Dental identification is extremely useful when attempting to achieve a positive identification or exclusion, either in ordinary cases of identification or in disaster victim identification (DVI) scenarios, where forensic odontology offers an expeditious and scientific method of comparative identification. The field of forensic odontology has experienced a significant change in the last thirty years, from first involving forensic odontologists only occasionally in identification cases, to them playing a key role in the identification process.
The definite establishment of the identity of a body essentially comes from a detailed comparison and matching of tangible ante mortem records and post mortem findings. It is rarely the case that the two match in all aspects, so some judgment is required. This often requires the application of logic, and unless the person dies on the day of their last dental treatment. Identification is established by comparison of known dental records of a missing person which is also known as antemortem data with dental findings from an unknown/ unidentified body, also known as post-mortem data. In human identification DNA, fingerprints, dental and unique medical records such as implants are admitted as scientific methods.

\section{Other Methods for Comparative Identification}

The frontal sinuses are two, in the posterior part of the superciliary arcs, and lies between the external and internal faces of the frontal bone. They are seldom symmetrical; generally, there is a septum between both, which usually deviates from the midline. The morphology may be visualized on the computed tomography and even posterio-anterior (PA) radiographs from 5 years of age. Thus, the reliability of comparing ante- and post-mortem radiographs of the frontal sinus for identification is well-founded. They have been found to have individual morphological variations. The first human identification through morphologic analysis of the frontal sinus to be accepted in a court case in the United States was described by Culbert and Law in $1927^{3}$

Cheiloscopy refers to the utilization of lip prints as means of personal identification in much the same manner as fingerprints. The pattern of wrinkles on the lips has individual characteristics as fingerprints. The wrinkles and grooves on the labial mucosa (called sulci labiorum) form a characteristic pattern called lip prints, the study of which is referred to as Cheiloscopy In 1971, Suzuki and Tsuchihashi conducted detailed research studying lip prints and they devised their classification system for cheiloscopy ${ }^{4}$ 
Rugae are the anatomical folds that are located on the anterior third of the palate behind the incisive papillae. They are also known as "Plica palatine," and the study of these patterns is called Palatoscopy. The palatal rugae like fingerprints do not change during the life of the individual $\&$, are protected from trauma and high temperatures for its internal position in the oral cavity, surrounded and protected by lips, cheeks, tongue, teeth, and bone, also prosthetic devices are not affected. It can be of special interest in edentulous cases and also in certain conditions where there are no fingers to be studied, such as burned bodies or bodies that underwent severe decomposition ${ }^{5}$. It is also interesting to note that some researchers have found rugae pattern to be specific to racial groups facilitating population identification. Thus the uniqueness, postmortem resistance, overall stability, and additionally low utilization cost makes palatal rugae an ideal forensic identification parameter ${ }^{6}$.

The identification of unknown or missing persons employing denture marking is also a successful method of identification in edentulous victims. Most dental identifications are based on restorations, caries, missing teeth, prosthetic devices such as partial and full removable prostheses which may be readily documented in the record. Labeled dentures can be used as unique identifiers to identify remains of edentulous victims or unlabelled dentures can be compared to or fitted on the casts of known patients when retained by the treating dentists or dental laboratories. Marked dentures can also be useful for patients residing in hospitals and community homes where dentures could be misplaced or mixed up when removed during sleep or for cleaning. ${ }^{7}$

\section{Role of Dental DNA in Identification}

DNA can be retrieved from dentin, cementum, periodontal ligament, alveolar bone, and pulpal tissue. Although the main source of DNA is blood but saliva, oral mucosal cells, and teeth are proven to be effective sources of DNA. Not only are they good sources, but DNA can be obtained in a non-invasive manner from buccal swabs as compared to drawing blood for comparison purposes. As teeth are resistant to immersion in water, decomposition, incineration (to certain limit), burial, etc. the hard tissues of teeth form a protective shield around the pulp tissue, providing preservation in high impact situations. It also helps in avoiding contamination during handling, however, for extracting DNA from dental pulp, the tooth must be intact and devoid of carious lesions, or endodontic treatments ${ }^{8}$

\section{Dental Age Estimation}

Gustafson, in 1950, pioneered in publishing a method for estimating the dental age of an adult ${ }^{9}$. In his paper, he researched six criteria, namely, attrition, secondary dentine deposition, cementum thickness, root resorption, root transparency, and periodontal attachment. Although Gustafson's technique is not employed anymore for forensic purposes, this technique paved the way for further research and development of techniques that are presently in use. It was found that when factors such as root transparency, secondary dentine deposition or attrition were used independently, they yielded much more accurate results as compared to a combination of all 6 criteria at the same time.

Lamendin ${ }^{10}$ developed a technique for age estimation in single-rooted teeth by measurements of 2 parameters, namely, loss of periodontal attachment and root transparency. Gustafson's technique and Lamendin's technique both can be employed only in deceased persons for age estimation as they require extracted teeth for assessment. Radiological methods were developed by various researchers such as Schour and Massler ${ }^{11}$, Gleiser and Hunt ${ }^{12}$, Demirjian ${ }^{13}$, Kvaal ${ }^{14}$, Cameriere ${ }^{15}$, for dental age estimation by studying various stages of mineralization of teeth during developmental years, open apices, analyzing the rate of secondary dentine deposition with age progression, etc.

\section{Reconstructive techniques in Identification}

Cases referred for reconstructive techniques of identification are frequently unknown human remains that have been rendered visually unidentifiable due to decomposition, skeletonization, or charring. Handling of charred remains can be a challenge as bones and teeth 
become brittle and susceptible to fracturing or even turning to ashes under the slightest pressure. As long as the body remains untouched the ash will maintain its 'tooth shape', but as soon as the body is moved all the ash will fall away and important information will be lost for good. A forensic odontologist at such scenes can stabilize these teeth and skull before the body is moved, ensuring the preservation of possibly vital identification evidence. Several materials have been used in the past to achieve this, and one such material is cold cure acrylic available at dental practices is suitable and can be carried to the scene for stabilizing and setting teeth and jaws. This then allows movement of the body without the risk of losing any of the teeth.

Techniques for reconstruction can be a useful aid in assisting the process of identification. Forensic facial reconstruction is one such technique that is used frequently in cold cases where the identity of skeletal remains is needed for the investigation to proceed. If it has not been possible to identify the remains when the profile data such as age, sex, race, stature have been explored, then the possibility of reconstruction of the face from the skull is considered. The purpose of reconstructing facial features is to trigger recognition by anyone known to the deceased, once a name can be put to the face, the identity is confirmed using a scientific primary identification technique of comparison of antemortem and post-mortem data such as fingerprints, dental, and DNA. It should be noted that the reasoning which governs the investigator's requisition for the reconstruction of facial features is that since there is no other way left to help identify the deceased, the process is worth trying, despite limitations.

Skull is the frame for the face to rest. Equipped with the current knowledge regarding skull anthropology, experienced experts within the field of craniofacial identification can reconstruct the face after studying the skull to a certain degree of resemblance with the face of an unidentified person in life. Overall, the technique is considered successful in aiding the investigation if certain features on the reconstructed face trigger recognition by someone known to the deceased. However, it cannot be claimed that an exact likeness of the person during life can be reproduced by this process, but current trends in research indicate that artificial intelligence and DNA technology can increase this accuracy.

After a thorough examination of the human remains, the investigator decides on a particular method for identification depending on the ante mortem and post mortem data available, information available on the database for reconstructive techniques. Craniofacial superimposition is performed if photos of the missing person are available to be superimposed and compared with the unidentified skull ${ }^{16}$. This technique of superimposition is used as a tertiary technique for identification unless accompanied by confirmatory dental identification. Craniofacial superimposition on its own can be used as an exclusion technique or to assess the possibility of a match in cases where a photograph of the missing person is available for comparison ${ }^{17}$. Craniofacial reconstruction is carried out in cases where there is no photograph or any other hint available regarding a missing person. Once the skull is mounted, photography is done to record the gross findings, including the extent of trauma to the skull or teeth before the commencement of examination. After adequate physical access to all aspects of the teeth and skull is secured, they should be thoroughly cleaned of any debris or decomposed material that may hinder adequate examination. Dental examination includes full mouth photography, odontogram charting and radiography. An issue that may be encountered during charting is clearly identifying tooth-coloured fillings. Current trends of minimally invasive dentistry and the exceptional colourmatching qualities of modern resin filling materials may present a challenge to forensic odontologist attempting to identify all tooth-coloured fillings. A portable LED light in the kit can come in handy, as the tooth-coloured fillings fluoresce when illuminated by wavelengths close to the ultraviolet (UV) range.

Once the photography is done, anthropological assessment of the skull is performed. Before attempting reconstruction, it is necessary to make a general assessment of the skull for age, race and sex. In addition, 
the build of the person and stature, may be ascertained from examination of the post-cranial skeleton and other associated findings such as clothing and footwear. Furthermore, it is essential to carry out a careful examination of the skull, looking for asymmetry and any features which may be sufficiently discriminatory which will assist in the reconstruction. A replica of the skull can be made by several methods. This has been traditionally done by taking impression of the original skull and then casting a replica in plaster or stone. It can also be done by $3 \mathrm{D}$ scanning and printing the skull digitally. Reconstruction is then performed on the replica prepared. Manchester method developed by Richard Neave is the most widely accepted technique for reconstruction, as it involves both anatomical and anthropometrical methods using soft tissue depth markers as guides for building the head and neck structure. Placement of soft tissue thickness markers allows precise placement of soft tissue on to the skull allowing the skull shape to determine the shape of reconstructed face. Once the markers are placed on the skull, it is easier to make adjustments and correct errors more effectively.

Facial reconstruction on human remains can be performed in a number of ways. It can be done completely by manual traditional methods of impression making, casting and then clay application. It also can be done by partially manual methods such as scanning and 3D printing the skull and then using clay for soft tissue build up. In recent times, completely digital methods have been used for facial reconstruction ${ }^{18}$

\section{Conclusion}

Identification of human remains is not only important for medico-legal purposes but also for humanitarian reasons. Unknown remains can be identified from anything that distinguishes them from another person. Bodies are routinely identified by secondary parameters such as tattoos, moles, scars, marks, or any other variation from normality. There is abundant literature and case reports regarding use of pathologies, asymmetries or other medical conditions to narrow down search for missing people. However, it should be brought to notice that forensic value of dental identifiers as primary parameter cannot be stressed enough. Dental anomalies, anatomical variations, presence of treatments, and unique oral and maxillofacial findings may be overlooked by the medical examiner. These variations, analysed by odontologists, often lead to a positive identification when comparative personal identification is performed.

Various factors influence whether the process of reconstructive identification will prove to be successful, one such factor is that the images of reconstructed face should reach people who knew the deceased during life. In some cases, it is possible that the relatives might not wish to come forward with information, especially, if they have been involved or are suspects. The chances of recognition would increase with media exposure. There are more chances of recognition if the deceased is from a small community with fewer reported missing people as compared to an unknown deceased in a big metropolis city. It is a fact that many people migrate to metropolitan cities for work or education opportunities. Often, their families are not aware that they have been missing for days or even months.

Reconstruction performed without knowledge and experience may not only lead to failure of identification but may also cause misidentification. Dearth in availability of soft tissue thickness data for Indian population leaves scope to further explore relationship between the hard and soft tissues of the skull, for increased accuracy in diverse Indian population. There is no doubt that techniques for facial reconstruction can be improved, with the advent of artificial intelligence and DNA technology can achieve higher precision than ever before.

Ethical Clearance- Not required for review article Source of Funding- Self funded

\section{Conflict of Interest - Nil}

\section{References}

1. Avon SL. Forensic odontology: the roles and responsibilities of the dentist, J Can Dent Assoc., 
2004, Jul-Aug; 70(7): 453-8

2. Keiser-Neilsen, S., Person Identification by Means of Teeth. John Wright \& Sons, Bristol, 1980

3. Suzanne Tanya Nethan., et al. "Frontal Sinus Dimensions: An Aid in Gender Determination". Acta Scientific Dental Sciences 2.12, 2018: 02-06.

4. Prabhu RV, Dinkar AD, Prabhu VD, Rao PK. Cheiloscopy: Revisited. Journal of Forensic Dental Sciences, 2012; 4:47-52.

5. Caldas I.M, Magalhaes T, Afonsa A. Establishing identity using chieloscopy and palatoscopy. Forensic Sci Int. 2007;165:1-9

6. Nayak P, Acharya AB, Padmini AT, Kaveri H. Differences in the palatal rugae shape in two populations of India. Arch Oral Biol. 2007;52:97782.

7. Johanson Gunnar, Ekman Bjorn. Denture Marking, Journal of American Dental Association, 1984, 3: 347-50

8. Muruganandhan J, Sivakumar G. Practical aspects of DNA-based forensic studies in dentistry. J Forensic Dent Sci. 2011;3:38-45.

9. Gustafson G. Age determination on teeth. J Am Dent Assoc. 1950;41:45-54

10. Lamendin H, Baccino E, Humbert JF, Tavernier JC, Nossintchouk RM, Zerilli A. A simple technique for age estimation in adult corpses: the two criteria dental method. J Forensic Sci. 1992;37:1373-9.

11. Schour I, Massler M; Development of human dentition. J Am Dent Assoc., 1941; 20: 379-427.

12. Gleiser I, Hunt EE (1955) The permanent mandibular first molar; its calcification, eruption and decay. Am J Phys Anthropol 13:253-284

13. Demirjian A, Goldstein H, Tanner JM. A new system of dental age assessment. Hum Biol. 1973;45:211-27

14. Kvaal SI, Kolltveit KM, Thomsen IO, Solheim T. Age estimation of adults from dental radiographs. Forensic SciInt. 1995;74:175-85

15. Cameriere R, Ferrante L, Cingolani M. Age estimation in children by measurement of open apices in teeth. Int J Legal Med 2006;120:49-52

16. Carvalho SPM, Silva RHA, Lopes C Jr, SalesPeres A. Use of images for human identification in forensic dentistry, Radiol Bras., 2009; 42: 125-30.

17. Iscan MY, Introduction of Techniques for Photographic Comparison: Potential and Problems. In: ISCAN, MY, Helmer RO (eds.) Forensic Analysis of the Skull: Craniofacial Analysis, Reconstruction, and Identification, 1993 New York, Wiley- Liss: 57-70. ISBN 0471560782

18. Wilkinson C. Computerized forensic facial reconstruction, Forensic Sci Med Pathol, 2005; 1: 173-7. 